\title{
Influence of Sleeping Habits on Adaptive Thermogenesis during Weight Loss in Adults
}

Jean-Philippe Chaput ${ }^{1 *}$, Caroline Y. Doyon ${ }^{2}$, Jessica McNeil ${ }^{3}$, Éric Doucet ${ }^{3}$ and Angelo Tremblay $^{2}$

${ }^{1}$ Healthy Active Living and Obesity Research Group, Children's Hospital of Eastern Ontario Research Institute, Ottawa, Ontario, Canada

${ }^{2}$ Department of Kinesiology, Faculty of Medicine, Laval University, Quebec City, Quebec, Canada

${ }^{3}$ School of Human Kinetics, Faculty of Health Sciences, University of Ottawa, Ottawa, Ontario, Canada

\begin{abstract}
Objective: To verify whether sleeping habits affect adaptive thermogenesis (i.e. greater than predicted decrease in resting energy expenditure, REE) in overweight and obese adults subjected to caloric restriction

Methods: A total of 123 overweight and obese men and women (mean \pm SD age, $41.1 \pm 6.0$ years; mean \pm SD body mass index, $\left.33.2 \pm 3.6 \mathrm{~kg} / \mathrm{m}^{2}\right)$ were tested before and $17.2 \pm 3.7$ weeks after dietary treatment $(-300 \mathrm{kcal} /$ day on average). Body fat mass (dual-energy X-ray absorptiometry), REE (indirect calorimetry) and sleep duration and quality (Pittsburgh Sleep Quality Index, PSQI) were assessed at both baseline and at the end of the weight loss program. Two sets of formula were used to predict changes in REE and the difference between the changes in the predicted REE from the reference equations and the changes in the measured REE were compared between sleep duration groups.

Results: The mean weight loss of all participants over the dietary intervention was $5.9 \pm 4.6 \mathrm{~kg}, 73 \%$ of which came from fat losses. The small dietary restriction led to a $57 \mathrm{kcal} /$ day reduction in REE at the end of the weight-loss program $(P<0.01)$. Using multivariable linear regression models, none of sleep duration or quality was associated with a greater than predicted decrease in REE. Similarly, adaptive thermogenesis was not significantly different between short- $(<6 \mathrm{~h} / \mathrm{night})$ and average-duration (7-9 h/night) sleepers and between poor (PSQI score $>5$ ) and good (PSQI score $\leq 5)$ sleepers.
\end{abstract}

Conclusion: This study provides evidence that sleeping habits are not associated with a greater than predicted decrease in REE during weight loss in adults exposed to small caloric restriction.

Keywords: Adaptive thermogenesis; Diet; Sleep; Weight loss

\section{Introduction}

One of the better documented effects of weight loss is that it is accompanied by a decrease in resting energy expenditure (REE) [1]. In fact, the evidence is accumulating in favor of adaptive modifications during weight loss that result in greater than expected decreases in REE than that which can be predicted through changes in fat mass (FM) and fat-free mass (FFM) [2]. Although controversy persists [3], there is increased recognition that adaptive thermogenesis in response to a negative energy balance may damper efforts to lose body fat [47]. Adaptive thermogenesis is a broad term applicable to increases or decreases of metabolic rate in response to a variety of different environmental factors. In the present paper, the term "adaptive thermogenesis" is defined as a greater than predicted decrease in REE after weight loss. In recent papers, we reported the quantitative importance of adaptive thermogenesis for weight-reduced obese individuals [8-10], which may in fact be more important than initially perceived.

The idea that adaptive thermogenesis could be influenced by environmental factors (such as the gut microbiota, infection with adenovirus 36 and sleep deprivation) has not been thoroughly addressed. Interestingly, organochlorine pollution seems to be a new factor affecting the control of thermogenesis in some obese individuals experiencing body weight loss [11]. In this previous study, we observed that increased plasma organochlorine concentration as a result of weight loss was the factor explaining the greatest proportion of the variance (47\%) in adaptive thermogenesis. More recently, we observed that sleeping habits can predict the magnitude of fat loss in adults exposed to moderate caloric restriction [12]. These results agree with those from Nedeltcheva et al. [13], who recently reported that insufficient sleep undermines dietary efforts to reduce adiposity. In their crossover study, 10 overweight adults were randomly assigned to sleep either $5.5 \mathrm{~h}$ or $8.5 \mathrm{~h}$ each night for 14 days in conjunction with moderate caloric restriction in a closed clinical research environment. Compared with participants who slept $8.5 \mathrm{~h}$ per night, participants who slept only $5.5 \mathrm{~h}$ lost less body fat and more fat-free body mass. Of note, the REE was significantly lower at the end of the 5.5 -h vs. 8.5-h time-in-bed condition and this decrease in absolute REE was greater than expected on the basis of the observed loss of fat and fat-free body mass alone [13]. A decreased REE after total sleep deprivation has also been previously reported [14]; however, experimentally-induced sleep deficit in the absence of caloric restriction does not seem to significantly impact REE [15-17].

In contrast, sleep deprivation has been shown to elevate metabolic rate and increase UCP1 gene expression in brown adipose tissue of rats $[18,19]$. However, the influence of sleeping habits on adaptive thermogenesis during weight loss is largely unknown in humans. The objective of the present study is to verify whether sleeping habits affect adaptive thermogenesis (i.e. greater than predicted decrease in REE)

*Corresponding author: Jean-Philippe Chaput, Healthy Active Living and Obesity Research Group, Children's Hospital of Eastern Ontario Research Institute, 401 Smyth Road, Ottawa, Ontario, Canada, K1H 8L1, Tel: +1 6137377600 ext. 3683; Fax: +1 613738 4800; E-mail: jpchaput@cheo.on.ca

Received February 17, 2012; Accepted March 19, 2012; Published March 23 2012

Citation: Chaput JP, Doyon CY, McNeil J, Doucet É, Tremblay A (2012) Influence of Sleeping Habits on Adaptive Thermogenesis during Weight Loss in Adults. Bioenerg Open Access 1:103. doi:10.4172/2167-76621000103

Copyright: (c) 2012 Chaput JP, et al. This is an open-access article distributed under the terms of the Creative Commons Attribution License, which permits unrestricted use, distribution, and reproduction in any medium, provided the original author and source are credited. 
in overweight and obese adults subjected to a small caloric restriction. We hypothesize that both short sleep duration ( $<6 \mathrm{~h}$ per night) and poor sleep quality (score $>5$ on the Pittsburgh Sleep Quality Index) at baseline are associated with a greater than predicted decrease in REE during weight loss in adults.

\section{Subjects and Methods}

\section{Subjects}

In order to maximize power, three weight loss studies performed in our facilities (Laval University, Quebec, Canada) have been pooled together. Details on these studies have been published elsewhere [2022]. Briefly, these weight-reducing interventions aimed at verifying the effect of either calcium + vitamin D supplementation, milk supplementation or a functional diet on body fat loss and various indicators of health. Only individuals involved in the control groups of these studies have been kept for statistical analyses in order to avoid the influence of the active ingredient on the main outcome measure (i.e. adaptive thermogenesis). Briefly, healthy overweight and obese men and women aged between 25 and 50 years were subjected to a weight loss intervention consisting of a small decrease in energy intake $(-300 \mathrm{kcal} /$ day on average), which was supervised by a dietician. The following inclusion criteria were also considered: absence of menopause (determined by the cessation of menstruation), stable body weight (body weight change $<3 \mathrm{~kg}$ for 2 months before intervention), $<3$ periods of 20 min of physical activity per week, no use of medication that could affect body weight, no smoking, normal blood pressure values $(<140 / 90 \mathrm{mmHg})$, consumption of $\leq 10$ alcoholic beverages per week, and consumption of $\leq 5$ cups of coffee per day. The length of the intervention varied between 15 and 24 weeks, depending on the study. Each participant met his/her assigned dietician every 2 weeks until the completion of the study. Compliance was assessed by comparing the diet prescribed (total daily energy intake and macronutrient composition) to the actual diet composition of the participants, the latter assessed every 2 weeks by means of $24 \mathrm{~h}$ food recalls. Study protocols were approved by the Laval University Ethics Committee. Written informed consent was obtained from all participants.

\section{Anthropometric and body composition measurements}

Height was measured to the nearest $0.1 \mathrm{~cm}$ using a standard stadiometer, and body weight was measured to the nearest $0.1 \mathrm{~kg}$ using a digital panel indicator scale (Beckman Industrial, Scotland, $\mathrm{UK})$. Body mass index (BMI) was calculated as body weight divided by height squared $\left(\mathrm{kg} / \mathrm{m}^{2}\right)$. Body FM and FFM were measured by dualenergy X-ray absorptiometry (GE Medical Systems Lunar, Diegem, Belgium). All measurements were performed according to standardized procedures at both baseline and at the end of the weight loss program.

\section{Measurement of resting energy expenditure}

REE was measured by indirect calorimetry after a $12 \mathrm{~h}$ overnight fast, as previously described in detail [23]. After a 15 min resting period, expired gas was collected through a mouthpiece for $15 \mathrm{~min}$ while the nose was clipped. Oxygen and carbon dioxide concentrations were determined by non-dispersive infrared analysis (Uras 10 E, Hartmann \& Braun, Frankfurt, Germany) whereas pulmonary ventilation was determined with a S-430A measurement system (KL Engineering, Ventura, CA, USA). The Weir formula [24] was used to determine the energy equivalent of oxygen volume. As previously reported [25], metabolic rate measurements as performed in our facilities provide a reliability coefficient of 0.9 and a coefficient of variation of less than $6 \%$.

\section{Calculation of predicted changes in resting energy expenditure}

Two sets of formula were used to predict changes in REE. First, the Harris-Benedict prediction equations [26] were used to determine whether the changes in REE by sleep duration group were greater than what was expected. The following equations were used:

$\operatorname{REE}(\mathrm{kcal} /$ day $)$ in men $=66.5+(13.75 \times$ weight in $\mathrm{kg})+(5.003 \times$ height in $\mathrm{cm})-(6.755 \times$ age in years $)$

$\operatorname{REE}(\mathrm{kcal} /$ day $)$ in women $=655.1+(9.563 \times$ weight in $\mathrm{kg})+(1.850$ $\times$ height in $\mathrm{cm})-(4.676 \times$ age in years $)$

Furthermore, a second formula was used from our previous work [23] which includes FM and FFM to predict REE before and after the weight loss intervention. The following equations were used:

$\mathrm{REE}(\mathrm{kcal} /$ day $)$ in men $=[1.28+(0.023 \times \mathrm{FM}$ in $\mathrm{kg})+(0.052 \times \mathrm{FFM}$ in $\mathrm{kg})] \times 344$

$\operatorname{REE}(\mathrm{kcal} /$ day $)$ in women $=[0.85+(0.016 \times \mathrm{FM}$ in kg $)+(0.059 \times$ FFM in $\mathrm{kg})] \times 344$

These reference equations were established from a control group of men $(n=112)$ and women $(n=166)$ of the same age group from the Quebec Family Study, as reported previously [23]. These equations have been shown to be equally applicable to the individuals of varying degrees of adiposity within the control population [23]. Changes in REE from baseline for the predicted and measured (actual) values were calculated for statistical analysis. Adaptive thermogenesis in this paper was considered as the difference between the changes in the predicted REE from the reference equations and the changes in the measured REE. In other words, adaptive thermogenesis represents here the greater than predicted decrease in REE induced by the weight-reducing program.

\section{Sleep assessment}

At baseline, each participant completed the Pittsburgh Sleep Quality Index (PSQI), a self-rated questionnaire that assesses sleep quality and disturbances over the preceding 1-month time interval [27]. Briefly, nineteen individual items generate seven component scores: subjective sleep quality, sleep latency, sleep duration, habitual sleep efficiency, sleep disturbances, use of sleeping medication, and daytime dysfunction. The sum of the component scores yields one total score with a maximum of 21 . A total PSQI score greater than 5 is highly sensitive and specific in distinguishing good from poor sleepers [27] and has been validated in a number of populations [28]. For the purpose of this study, both sleep quality (total PSQI score) and sleep duration (self-reported from the PSQI) were used for statistical analyses. Data were analyzed as continuous and with the use of categories for sleep length (short-duration sleepers, $<6 \mathrm{~h}$ per night vs. average-duration sleepers, 7-9 h per night) and sleep quality (poor sleepers, PSQI score $>5$ vs. good sleepers, PSQI score $\leq 5$ ), as previously reported [29,30].

\section{Diet assessment}

In addition to assess the compliance of the participants every 2 weeks by means of $24 \mathrm{~h}$ food recalls, diet was evaluated with a 3-day food record, including 2 weekdays and 1 weekend day, at baseline and at the end of the intervention. Participants were shown how to complete the record by a dietician, who provided instructions about measuring the quantities of ingested foods. This method of dietary assessment has been shown to provide a relatively reliable measure of diet in this population [31]. However, self-reported energy intakes should be interpreted with caution because of well-known bias and 
Citation: Chaput JP, Doyon CY, McNeil J, Doucet É, Tremblay A (2012) Influence of Sleeping Habits on Adaptive Thermogenesis during Weight Loss in Adults. Bioenerg Open Access 1:103. doi:10.4172/2167-7662.1000103

imprecision [32,33]. Mean daily energy intake was estimated by a dietician using a computerized version of the Canadian Nutrient File [34]. The change in total energy intake from baseline (in kcal/day) was used in the analyses as a covariate.

\section{Statistical analysis}

Sleep quantity (h/day) and quality (PSQI score) at baseline were used to predict adaptive thermogenesis (i.e. difference between the changes in the predicted REE from the reference equation and the changes in the measured REE) using multivariable linear regression analyses. The models were adjusted for age, sex, baseline BMI, length of the intervention and the change in energy intake as covariates. Paired $t$ tests were also performed to compare predicted and measured changes in REE between sleep duration groups. All regression models and $t$ tests were tested for sex interactions (sex $\times$ sleeping habits). Overall, there were no significant sex interactions; therefore, the results are presented with both sexes combined to maximize power. A two-tailed $P$ value of less than 0.05 was considered to indicate statistical significance. Data are presented as mean values $\pm \mathrm{SD}$. All statistical analyses were performed using the JMP version 9 program (SAS Institute, Cary, NC).

\section{Results}

Characteristics of subjects participating in the study are shown in Table 1. Almost all variables showed significant differences; however, a full discussion of the effects of the weight-loss program on the overall physiological constitution is beyond the scope of this paper. A total of 70 men and 53 women, aged $41.2 \pm 5.7$ and $40.9 \pm 6.4$ years respectively, were investigated before and $17.2 \pm 3.7$ weeks after dietary treatment. The mean weight loss of all participants over the dietary intervention was $5.9 \pm 4.6 \mathrm{~kg}, 73 \%$ of which came from fat stores. The small dietary restriction ( $-300 \mathrm{kcal} /$ day) led to a $57 \mathrm{kcal} /$ day reduction in REE at the end of the weight-loss program $(P<0.01)$. The actual decrease in REE $(-57 \mathrm{kcal} /$ day) was not significantly different than what was predicted using either the Harris-Benedict [26] (-85 kcal/day) or the Doucet et al. [23] $(-64 \mathrm{kcal} / \mathrm{d})$ prediction equation. The $24-\mathrm{h}$ food recalls showed that participants complied at $\geq 90 \%$ with their energyrestricted diet, as assessed by the difference between total energy intake actually consumed throughout the program and the prescribed dietary prescription.

\begin{tabular}{|l|c|c|}
\hline & Before & After \\
\hline Body weight (kg) & $94.7 \pm 14.5$ & $88.8 \pm 13.7^{*}$ \\
\hline BMI (kg/m2) & $33.2 \pm 3.6$ & $31.3 \pm 3.4^{*}$ \\
\hline Fat mass (kg) & $35.7 \pm 8.3$ & $31.4 \pm 7.9^{*}$ \\
\hline Fat mass (\%) & $38.1 \pm 7.1$ & $35.6 \pm 7.1^{*}$ \\
\hline Fat-free mass (kg) & $59.0 \pm 5.2$ & $57.4 \pm 4.7^{* *}$ \\
\hline Sleep duration (h) & $7.1 \pm 1.0$ & $7.4 \pm 1.2$ \\
\hline PSQl (total score) & $4.78 \pm 2.83$ & $3.88 \pm 2.72^{*}$ \\
\hline Energy intake (kcal/d) & $1253 \pm 485$ & $976 \pm 385^{*}$ \\
\hline REE (kcal/d) & $1804 \pm 299$ & $1747 \pm 284^{*}$ \\
\hline Adaptive thermogenesis (kcal/d) ${ }^{\text {a }}$ & & \\
\hline Harris-Benedict formula [26] & & $28.0 \pm 102$ \\
\hline Doucet et al. formula [23] & & $7.2 \pm 99$ \\
\hline
\end{tabular}

BMI: Body Mass Index; PSQI: Pittsburgh Sleep Quality Index; REE: Resting Energy Expenditure.

Values are mean \pm SD.

aDifference between the changes in the predicted REE from the reference equation and the changes in the measured REE.

${ }^{*} P<0.01$ vs. before the intervention.

${ }^{* *} P<0.05$ vs. before the intervention.

$n=123$

Table 1: Characteristics of participants before and after the dietary intervention.
Using multivariable linear regression models, sleep duration and quality were not associated with adaptive thermogenesis (Table 2). More specifically, the difference between the changes in the predicted REE from the reference equation and the changes in the measured REE were not significantly different between short- $(<6 \mathrm{~h} /$ night $)$ and average-duration (7-9 h/night) sleepers (Table 3) and between poor (PSQI score $>5$ ) and good (PSQI score $\leq 5$ ) sleepers (Table 4). Although absolute REE tended to be higher in short-duration sleepers compared to average-duration sleepers because of their higher body mass (Table 3 ), this difference was not significantly different in relative terms (i.e. $\mathrm{REE} / \mathrm{kg}$ of body weight). Of note, analysis of our data using masscorrected REE (i.e. REE/kg of body weight) instead of total REE yielded the same results (data not shown).

\section{Discussion}

Collectively, we observed that both total sleep time (h/night) and sleep quality (PSQI score) were not predictive of a greater than

\begin{tabular}{|c|c|c|c|}
\hline & \multicolumn{3}{|c|}{ Adaptive thermogenesis (kcal/day) } \\
\hline & $\beta$ & $r$ & $P$ \\
\hline \multicolumn{4}{|c|}{ Harris-Benedict prediction equation [26] } \\
\hline \multicolumn{4}{|c|}{ Sleep duration (h/night) } \\
\hline Unadjusted model & -20.71 & 0.14 & 0.29 \\
\hline Adjusted model $^{\mathrm{b}}$ & -24.7 & 0.16 & 0.22 \\
\hline \multicolumn{4}{|c|}{ Sleep quality (PSQI score) } \\
\hline Unadjusted model & -0.49 & 0.01 & 0.93 \\
\hline Adjusted model ${ }^{\mathrm{b}}$ & 1.48 & 0.03 & 0.79 \\
\hline \multicolumn{4}{|c|}{ Doucet et al. prediction equation [23] } \\
\hline \multicolumn{4}{|c|}{ Sleep duration (h/night) } \\
\hline Unadjusted model & -20.87 & 0.13 & 0.32 \\
\hline Adjusted model ${ }^{\mathrm{b}}$ & -28.14 & 0.17 & 0.19 \\
\hline \multicolumn{4}{|c|}{ Sleep quality (PSQI score) } \\
\hline Unadjusted model & -1.66 & 0.03 & 0.77 \\
\hline Adjusted model $^{\mathrm{b}}$ & 0.90 & 0.02 & 0.87 \\
\hline
\end{tabular}

PSQI: Pittsburgh Sleep Quality Index

adaptive thermogenesis is defined here as the difference between the changes in the predicted resting energy expenditure from the reference equation and the changes in the measured resting energy expenditure.

${ }^{b}$ Model adjusted for age, sex, baseline body mass index, length of the intervention and the change in energy intake.

$n=123$

Table 2: Multivariable linear regression models for the associations between sleeping habits and adaptive thermogenesis ${ }^{a}$.

\begin{tabular}{|c|c|c|c|c|}
\hline & \multicolumn{2}{|c|}{$\begin{array}{l}\text { Short-duration sleepers } \\
(<6 \mathrm{~h} / \text { night })\end{array}$} & \multicolumn{2}{|c|}{$\begin{array}{l}\text { Average-duration sleepers } \\
\text { (7-9 h/night) }\end{array}$} \\
\hline & Before & After & Before & After \\
\hline \multicolumn{5}{|l|}{ Harris-Benedict formula (26) } \\
\hline Predicted REE (kcal/d) & $1867 \pm 262$ & $1837 \pm 265$ & $1727 \pm 303^{* *}$ & $1614 \pm 237^{*}$ \\
\hline Measured REE (kcal/d) & $1801 \pm 321$ & $1829 \pm 281$ & $1692 \pm 297$ & $1621 \pm 256^{*}$ \\
\hline $\begin{array}{l}\text { Adaptive thermogenesis } \\
(\mathrm{kcal} / \mathrm{d})^{\mathrm{a}}\end{array}$ & & $2 \pm 91$ & & $42 \pm 98$ \\
\hline \multicolumn{5}{|l|}{ Doucet et al. formula [23] } \\
\hline Predicted REE (kcal/d) & $1725 \pm 253$ & $1718 \pm 232$ & $1598 \pm 268^{* \star}$ & $1505 \pm 229^{*}$ \\
\hline Measured REE (kcal/d) & $1801 \pm 321$ & $1829 \pm 281$ & $1692 \pm 297$ & $1621 \pm 256^{*}$ \\
\hline $\begin{array}{l}\text { Adaptive thermogenesis } \\
(\mathrm{kcal} / \mathrm{d})^{\mathrm{a}}\end{array}$ & & $21 \pm 86$ & & $23 \pm 89$ \\
\hline
\end{tabular}

REE: Resting Energy Expenditure

Values are mean \pm SD.

aDifference between the changes in the predicted REE from the reference equation and the changes in the measured REE.

Data on adaptive thermogenesis are not significantly different between sleep duration groups (paired $t$ test).

${ }^{*} P<0.01$ vs. short-duration sleepers after the intervention.

${ }^{* *} P<0.05$ vs. short-duration sleepers before the intervention.

$n=24$ (short-duration sleepers) and $\mathrm{n}=91$ (average-duration sleepers)

Table 3: Predicted and measured resting energy expenditure before and after the dietary intervention. 


\begin{tabular}{|c|c|c|c|c|}
\hline & \multicolumn{2}{|c|}{$\begin{array}{l}\text { Poor sleepers (PSQI } \\
\text { score >5) }\end{array}$} & \multicolumn{2}{|c|}{$\begin{array}{l}\text { Good sleepers (PSQI } \\
\text { score } \leq 5 \text { ) }\end{array}$} \\
\hline & Before & After & Before & After \\
\hline \multicolumn{5}{|c|}{ Harris-Benedict formula [26] } \\
\hline Predicted REE (kcal/d) & $1830 \pm 303$ & $1778 \pm 300$ & $1847 \pm 298$ & $1749 \pm 269$ \\
\hline Measured REE (kcal/d) & $1750 \pm 298$ & $1735 \pm 237$ & $1816 \pm 301$ & $1739 \pm 301$ \\
\hline $\begin{array}{l}\text { Adaptive thermogenesis } \\
(\mathrm{kcal} / \mathrm{d})^{\mathrm{a}}\end{array}$ & & $37 \pm 96$ & & $21 \pm 101$ \\
\hline \multicolumn{5}{|l|}{ Doucet et al. formula [23] } \\
\hline Predicted REE (kcal/d) & $1681 \pm 285$ & $1659 \pm 280$ & $1716 \pm 263$ & $1633 \pm 245$ \\
\hline Measured REE (kcal/d) & $1750 \pm 298$ & $1735 \pm 237$ & $1816 \pm 301$ & $1739 \pm 301$ \\
\hline $\begin{array}{l}\text { Adaptive thermogenesis } \\
(\mathrm{kcal} / \mathrm{d})^{\mathrm{a}}\end{array}$ & & $7 \pm 98$ & & $6 \pm 109$ \\
\hline
\end{tabular}

PSQI: Pittsburgh Sleep Quality Index; REE: Resting Energy Expenditure

Values are mean $\pm S D$

aDifference between the changes in the predicted REE from the reference equation and the changes in the measured REE.

Data on adaptive thermogenesis are not significantly different between sleep duration groups (paired t test).

$n=40$ (poor sleepers) and $n=83$ (good sleepers).

Table 4: Predicted and measured resting energy expenditure before and after the dietary intervention.

expected decrease in REE after body weight loss in adults. These results are novel and suggest that sleeping habits do not influence the adaptive component of thermogenesis in a context of small caloric restriction leading to modest weight loss. Future studies should put efforts in determining whether sleeping habits impact adaptive thermogenesis in individuals subjected to a more severe dietary restriction.

The $57 \mathrm{kcal} /$ day decrease in REE observed in this study appears to be lower than what was recently reported (i.e. $90 \mathrm{kcal} / \mathrm{day}$ ) from the results of a systematic review evaluating the relative changes in REE during weight loss [1]. A decrease of REE relative to weight loss of -15.4 $\mathrm{kcal} / \mathrm{kg}$ was observed from 2996 subjects and a greater drop in REE was observed for short interventions (more than 2 but less than 6 weeks) when compared with long interventions ( $>6$ weeks). Along the same lines, we observed an adaptive thermogenesis of $28 \mathrm{kcal} /$ day in the whole sample with the use of the Harris-Benedict prediction equation. This value of adaptive thermogenesis is somewhat smaller compared to what has recently been published (i.e. $45.6 \mathrm{kcal} /$ day) in a systematic review aimed at quantifying the greater than predicted decrease in REE during weight loss [2]. This discrepancy can be explained by many factors, including the magnitude of the energy restriction, the length of the intervention, the proportion of FFM lost, and the many interindividual differences. The high inter-individual variability in response to the weight-loss intervention in the present study (mean weight loss of $5.9 \pm 4.6 \mathrm{~kg}$ ) despite good compliance suggests that many factors can impact the success of a weight-reducing program. Thus, the smaller than expected decrease in REE after weight loss observed in our study is a positive outcome and we observed that sleeping habits do not seem to significantly impact this component of energy expenditure.

Our results are somewhat in contrast to recent findings showing that REE was significantly lower at the end of a short-term sleep restriction intervention compared to an 8.5 -h time-in-bed condition [13]. Although a clear advantage of laboratory studies is the very accurate control of environmental parameters, the tightly controlled sleep laboratory setting that was used in their crossover study is very different compared to the present observational study. In addition, the severity of sleep restriction (usually $4 \mathrm{~h} /$ night) in these short-term intervention studies is not representative of the real-life conditions of short-duration sleepers who are generally not able to tolerate such a severe sleep deprivation on a chronic basis. In their study, Nedeltcheva et al. [13] observed that the proportion of body weight lost as fat during the sleep restriction condition was only 25\% (as opposed to $73 \%$ in the present study). These considerable catabolic effects of sleep deprivation are much less common in a real-life setting and can help explain the decrease in REE [35]. Thus, the observed differences between our findings and those from Nedeltcheva et al. [13] can readily be explained by the different study designs used and by the severity of sleep restriction.

It has been reported that prescribing dietary interventions to obese, short-duration sleepers might be counterproductive as most of them already have higher hunger levels [36]. Moreover, lack of sleep has been reported in some studies to decrease plasma leptin levels, increase plasma ghrelin and cortisol levels, alter glucose homeostasis and activate the orexin system, all of which impact the control of appetite and might compromise the efficacy of dietary interventions [37]. In the present naturalistic study, the compliance of participants was as good in poor as in good sleepers. This finding suggests that the small caloric restriction used in our study might have been well tolerated by the participants, independently of their sleeping habits.

It is well known that thermogenesis in humans is largely a function of sympathetic nervous system activity [38] and sympathetic nervous system has been shown to decrease in response to weight loss [39]. Interestingly, lack of sleep is a stressor for the human organism and is associated with an increased sympathetic tone [40]. Although speculative, this adaptation might help counteract the greater than expected decrease in REE in response to weight loss in short-duration sleepers. However, more studies are needed to better quantify the influence of sleeping habits on adaptive thermogenesis and to better document the underlying mechanisms.

The present study has some limitations that need to be taken into consideration before generalizing our study findings. Firstly, the small sample size and preliminary nature of this investigation precludes any definitive conclusion. For example, it is unknown for the moment whether a larger energy restriction would have more impact on adaptive thermogenesis among poor sleepers. Furthermore, the data for this study have been gathered from 3 different studies and therefore limits the potential to analyze the participants in more detail. However, we hope that this study will generate larger and well-designed studies to address the effects of poor sleeping habits on adaptive thermogensis in human. Secondly, we have to keep in mind the well-known limitations of questionnaire-based measurements (e.g. sleep duration and diet assessment) as well as the possibility of residual confounding factors. Finally, the present study is only suited for the identification of associations and does not permit causal inferences.

In summary, our study provides evidence to the effect that sleeping habits are not associated with a greater than predicted decrease in REE during weight loss in adults exposed to a small caloric restriction, either with the use of total REE or mass-corrected REE. Future studies should verify whether a more severe dietary restriction would give the same outcome.

\section{Acknowledgements}

We express our gratitude to the subjects for their participation and the staff of the Physical Activity Sciences Laboratory at Laval University for their contribution to this study. None of the authors had a personal interest or potential conflict of interest with the organizations sponsoring the projects. Funding to conduct these studies was obtained from Wyeth Consumer Healthcare Inc., the Dairy Farmers of Canada, the US National Dairy Council and the Canadian Institutes of Health Research. JPC holds a Junior Research Chair in Healthy Active Living and Obesity Research. ED is a recipient of a CIHR/Merck-Frosst New Investigator Award, CFI/ OIT New Opportunities Award and of an Early Research Award. AT is partly funded by the Canada Research Chair in Environment and Energy Balance. 
Citation: Chaput JP, Doyon CY, McNeil J, Doucet É, Tremblay A (2012) Influence of Sleeping Habits on Adaptive Thermogenesis during Weight Loss in Adults. Bioenerg Open Access 1:103. doi:10.4172/2167-7662.1000103

\section{Disclosure Statement}

The authors declare no conflict of interest.

\section{References}

1. Schwartz A, Doucet $E$ (2010) Relative changes in resting energy expenditure during weight loss: a systematic review. Obes Rev 11: 531-547.

2. Schwartz A, Kuk JL, Lamothe G, Doucet E (2012) Greater Than Predicted Decrease in Resting Energy Expenditure and Weight Loss: Results from a Systematic Review. Obesity (Silver Spring).

3. Flatt JP (2007) Exaggerated claim about adaptive thermogenesis. Int J Obes (Lond) 31: 1626; author reply 1627-1628.

4. Rosenbaum M, Leibel RL (2010) Adaptive thermogenesis in humans. Int $J$ Obes 34: S47-S55.

5. Wijers SL, Saris WH, van Marken Lichtenbelt WD (2009) Recent advances in adaptive thermogenesis: potential implications for the treatment of obesity. Obes Rev 10: 218-226.

6. Goele K, Bosy-Westphal A, Rumcker B, Lagerpusch M, Muller MJ (2009) Influence of changes in body composition and adaptive thermogenesis on the difference between measured and predicted weight loss in obese women. Obes Facts 2: 105-109.

7. Tremblay A, Chaput JP (2009) Adaptive reduction in thermogenesis and resistance to lose fat in obese men. Br J Nutr 102: 488-492.

8. Tremblay A, Royer MM, Chaput JP, Doucet E (2012) Adaptive thermogenesis can make a difference in the ability of obese individuals to lose body weight. Int J Obes (in press)

9. Tremblay A, Major GC, Doucet E, Trayhurn P, Astrup A (2007) Role of adaptive thermogenesis in unsuccessful weight-loss intervention. Future Lipidol 2: 651 658

10. Major GC, Doucet E, Trayhurn P, Astrup A, Tremblay A (2007) Clinical significance of adaptive thermogenesis. Int J Obes (Lond) 31: 204-212.

11. Tremblay A, Pelletier C, Doucet E, Imbeault P (2004) Thermogenesis and weight loss in obese individuals: primary association with organochlorine pollution. Int J Obes Relat Metab Disord 28: 936-939.

12. Chaput JP, Tremblay A (2012) Sleeping habits predict the magnitude of fat loss in adults exposed to moderate caloric restriction. Obes Facts (in press).

13. Nedeltcheva AV, Kilkus JM, Imperial J, Schoeller DA, Penev PD (2010) Insufficient sleep undermines dietary efforts to reduce adiposity. Ann Intern Med 153: 435-441.

14. Benedict C, Hallschmid M, Lassen A, Mahnke C, Schultes B, et al. (2011) Acute sleep deprivation reduces energy expenditure in healthy men. Am J Clin Nutr 93: $1229-1236$

15. Hursel R, Rutters F, Gonnissen HK, Martens EA, Westerterp-Plantenga MS (2011) Effects of sleep fragmentation in healthy men on energy expenditure, substrate oxidation, physical activity, and exhaustion measured over $48 \mathrm{~h}$ in a respiratory chamber. Am J Clin Nutr 94: 804-808.

16. Nedeltcheva AV, Kilkus JM, Imperial J, Kasza K, Schoeller DA, et al. (2009) Sleep curtailment is accompanied by increased intake of calories from snacks. Am J Clin Nutr 89: 126-133.

17. Bosy-Westphal A, Hinrichs S, Jauch-Chara K, Hitze B, Later W, et al. (2008) Influence of partial sleep deprivation on energy balance and insulin sensitivity in healthy women. Obes Facts 1: 266-273

18. Koban M, Swinson KL (2005) Chronic REM-sleep deprivation of rats elevates metabolic rate and increases UCP1 gene expression in brown adipose tissue. Am J Physiol Endocrinol Metab 289: E68-E74.

19. Cirelli C, Tononi G (2004) Uncoupling proteins and sleep deprivation. Arch Ita Biol 142: 541-549.

20. Major GC, Alarie F, Doré J, Phouttama S, Tremblay A (2007) Supplementation with calcium + vitamin $D$ enhances the beneficial effect of weight loss on plasma lipid and lipoprotein concentrations. Am J Clin Nutr 85: 54-59.

21. Gilbert JA, Joanisse DR, Chaput JP, Miegueu P, Cianflone K, et al. (2011) Milk supplementation facilitates appetite control in obese women during weight loss: a randomized, single-blind, placebo-controlled trial. Br J Nutr 105: 133-143.

22. Drapeau V, Arguin H, Tremblay A (2010) Impact of a satiating diet on satiety quotient and body weight in obese men. Appl Physiol Nutr Metab 35: 373.

23. Doucet E, St-Pierre S, Alméras N, Després JP, Bouchard C, et al. (2001) Evidence for the existence of adaptive thermogenesis during weight loss. $\mathrm{Br}$ J Nutr 85: 715-723

24. Weir JB (1949) New method for calculating metabolic rate with specia references to protein metabolism. J Physiol 109: 1-9.

25. Bouchard C (1985) Body-composition assessments in youth and adults. In Roche Jr E, editor. Report of the Sixth ROSS Conference on Medical Research Ross Laboratories, Columbus, $\mathrm{OH}$.

26. Harris JA, Benedict FG (1918) A biometric study of human basal metabolism Proc Natl Acad Sci U S A 4: 370-373.

27. Buysse DJ, Reynolds CF 3rd, Monk TH, Berman SR, Kupfer DJ (1989) The Pittsburgh Sleep Quality Index: a new instrument for psychiatric practice and research. Psychiatry Res 28: 193-213.

28. Carpenter JS, Andrykowski MA (1998) Psychometric evaluation of the Pittsburgh Sleep Quality Index. J Psychosom Res 45: 5-13.

29. Chaput JP, Després JP, Bouchard C, Tremblay A (2011) The association between short sleep duration and weight gain is dependent on disinhibited eating behavior in adults. Sleep 34: 1291-1297.

30. Chaput JP, Leblanc C, Pérusse L, Després JP, Bouchard C, et al. (2009) Risk factors for adult overweight and obesity in the Quebec Family Study: have we been barking up the wrong tree? Obesity (Silver Spring) 17: 1964-1970.

31. Tremblay A, Sévigny J, Leblanc C, Bouchard C (1983) The reproducibility of a three-day dietary record. Nutr Res 3: 819-830.

32. Lansky D, Brownell KD (1982) Estimates of food quantity and calories: errors in self-report among obese patients. Am J Clin Nutr 35: 727-732.

33. Schoeller DA (1995) Limitations in the assessment of dietary energy intake by self-report. Metabolism 44: 18-22.

34. Government of Canada (2005) The Canadian Nutrient File. Health and Welfare Canada, Ottawa, Canada.

35. Scrimshaw NS, Habicht JP, Pellet P, Piché ML, Cholakos B (1966) Effects of sleep deprivation and reversal of diurnal activity on protein metabolism of young men. Am J Clin Nutr 19: 313-319.

36. Spiegel K, Tasali E, Penev P, Van Cauter E (2004) Brief communication: Sleep curtailment in healthy young men is associated with decreased leptin levels, elevated ghrelin levels, and increased hunger and appetite. Ann Intern Med 141: 846-850.

37. Leproult R, Van Cauter E (2010) Role of sleep and sleep loss in hormona release and metabolism. Endocr Dev 17: 11-21.

38. Saris WH (1995) Effects of energy restriction and exercise on the sympathetic nervous system. Int J Obes Relat Metab Disord 19: S17-S23.

39. Aronne LJ, Mackintosh R, Rosenbaum M, Leibel RL, Hirsch J (1995) Autonomic nervous system activity in weight gain and weight loss. Am J Physiol 269: R222-R225.

40. Balbo M, Leproult R, Van Cauter E (2010) Impact of sleep and its disturbances on hypothalamo-pituitary-adrenal axis activity. Int $\mathrm{J}$ Endocrinol.

Submit your next manuscript and get advantages of OMICS Group submissions

Unique features:

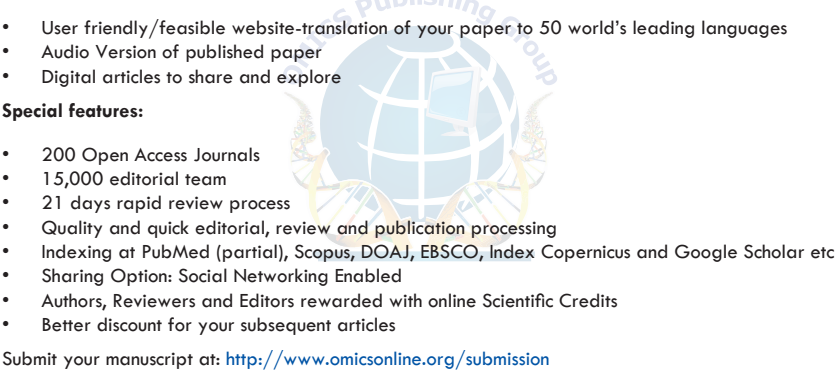

